\title{
What happens with MUST scores?
}

\author{
C. A. Cooper, E. R. Brierley, E. Rowlands and S. Burden \\ Dietetic Department, Manchester Royal Infirmary, Oxford Road, Manchester M13 9WL, UK
}

It is recommended that all patients are screened for malnutrition on admission to hospital. Following screening, a suitable care plan should be available outlining nutrition support interventions appropriate to the level of malnutrition risk identified ${ }^{(1,2)}$.

The aim of this study was to evaluate the Integrated Care Plan (ICP) used for the Malnutrition Universal Screening Tool (MUST), in a large teaching hospital, involving ward staff in a developmental project to improve the delivery of basic nutritional care initiated after screening.

An audit of 100 patients was conducted to determine if MUST was completed correctly and if basic nutritional support actions were completed as a result of screening. Data were collected from the MUST ICP, nursing, medical and dietetic records. Eight focus groups were conducted, 6 involving 22 nurses and another 2 involving 10 dietitians and patients. Qualitative data from the focus groups were transcribed verbatim and analysed using thematic analysis in NVivo8.

Screening tools were completed correctly for height in 93 cases, for weight in 69 cases and for usual body weight in 83 cases. Ward staff and dietitians scores were compared and scores were the same for 92 patients (level of agreement Kappa $=0.82$ ). The ICP was redesigned and qualitative data from the focus groups were substantiated by discussing the new care plan with ward staff.

\begin{tabular}{lcc}
\hline & Score $1, n=9(\%)$ & Score 2, $n=21(\%)$ \\
\hline Has daily care plan been followed & $7(77)$ & $6(28)$ \\
Has patient been referred to dietitian & $5(55)$ & $2(9)$ \\
Have food charts been completed & $2(22)$ & $7(33)$ \\
Have supplements been given & $2(22)$ & $7(33)$ \\
Have snacks been provided & $2(22)$ & $7(33)$ \\
\hline
\end{tabular}

\begin{tabular}{l}
\hline Key themes from the focus group data \\
Repetition of information \\
One document covering all nutritional aspects, including 3-day food chart and Red Tray \\
Equal amounts of weekly weights and MUST scores, as well as daily care plan \\
Variance at the bottom of the daily care plan and not on a separate page \\
Stepwise written instructions on how to complete MUST, using tick boxes and initials \\
Not audited, therefore not thought of as important document to complete \\
Assistance and snacks given to patients but not documented on ICP; written in Kardex \\
\hline
\end{tabular}

In this Trust, MUST was completed correctly in the majority of patients; however, the daily care plan was poorly followed. Focus groups engaged ward staff in the re-design of the ICP, to make this more user friendly. The aim is to improve compliance with the ICP and increase implementation of basic nutritional care following screening at the ward level.

1. Elia M, for the Malnutrition Advisory Group (2003) The MUST Report. Nutritional Screening of Adults: A Multidisciplinary Responsibility. British Association for Parenteral and Enteral Nutrition.

2. National Institute for Clinical Excellence (2006) Nutritional support for adults: oral nutrition support, enteral tube feeding and parenteral nutrition. 